\title{
CORRIGENDUM
}

\section{Text input speed in persons with cervical spinal cord injury}

\author{
S Pouplin, N Roche, I Vaugier, S Cabanilles, C Hugeron and D Bensmail
}

Spinal Cord (2016) 54, 490; doi:10.1038/sc.2016.21

Correction to: Spinal Cord (2016) 54, 158-162; doi: 10.1038/ sc.2015.147; published online 15 September 2015

Since the publication of this article, the authors have noticed an error in the results section of the abstract and main text. The correct sentence in the abstract is:

'Thirty-five participants with cervical SCI and 21 able-bodied people were included. Median TIS of participants with cervical SCI was
$11(6 ; 14)$ words per minute (w.p.m.) and of able-bodied participants was 19 (14; 24) w.p.m. $(P=0.001)$. Median TIS of participants with lesions at or above $\mathrm{C} 5$ was $12(4 ; 13)$ w.p.m. and of those with lesions below C5 was 10 (9; 18) w.p.m. $(P=0.38)$.'

The correct sentence in the main text under 'Text input speed' is: 'Median TIS was $11(6 ; 14)$ w.p.m. for participants with cervical SCI and $19(14 ; 24)$ w.p.m. for able-bodied participants $(P=0.001)$.'

The authors apologise for any inconvenience caused. 